\title{
Parkinsonism in Type I Gaucher's Disease
}

\author{
Kaori Itokawa ${ }^{1}$, Naotoshi Tamura ${ }^{1}$, Nobutaka Kawai ${ }^{2}$, Kunio Shimazu ${ }^{1}$ and Kenji Ishii ${ }^{3}$
}

\begin{abstract}
The majority of patients with type I Gaucher's disease never develop neurological signs or symptoms. However, several case reports of Parkinson's disease associated with type I Gaucher's disease have been published, suggesting a genetic link between the two diseases. Hence, detailed clinical investigations are required when the two diseases occur simultaneously, in order to determine whether this is coincidental or whether a true association is present. We present a Japanese man in whom parkinsonism was associated with type I Gaucher's disease. Findings of brain positron tomography (PET) and metaiodobenzylguanidine (MIBG) cardiac scintigraphy are presented.
\end{abstract}

Key words: Gaucher's disease, parkinsonism, metaiodobenzylguanidine myocardial SPECT, fluorodeoxyglucose PET, levodopa

(DOI: 10.2169/internalmedicine.45.1790)

\section{Case Report}

A 42-year-old man with type I Gaucher's disease was admitted to our hospital with an 8-month history of poor mobility of the right leg. His intelligence seemed normal; he was working in computer engineering with no reported difficulties. Gaucher's disease had been diagnosed at the age of 31 , based on the following typical findings; hepatosplenomegaly, Gaucher cells on bone marrow biopsy, reduction in $\beta$-glucosidase activity, and L444P/R463C mutation of the glucocerebrosidase gene on genetic analysis. Enzyme replacement therapy had been started at the age of 34 , and signs of Gaucher's disease, including anemia and hepatosplenomegaly, were absent at the present admission. On neurological examination, cogwheel-type rigidity was evident in the right upper and lower limbs, and poor arm swing was noted on walking, but tremor was absent. Mental function was normal. However, his speech was limited and monotonous, and facial expression was masked. Finger tapping was slow on the right side but retropulsion was absent. Deep tendon reflexes were normal and pathological reflexes were absent. Dysdiadochokinesis was absent. Brain MRI failed to demonstrate any lesions. PET using [F-18] fluorodeoxyglucose (FDG) as a tracer showed hypometabolism in the frontal and parietal cortices, although metabolism in the striatum was well preserved (Fig. 1). [I-123] MIBG myocardial SPECT revealed persistent low uptake in the inferoposterior area on early phase and complete absence of MIBG uptake in this area on delayed phase. [Tc-99m] tetrofosmin SPECT revealed low uptake in the septum and inferior areas (Fig. 2), suggesting that the reduced MIBG uptake in these areas was due to old myocardial infarction. However, ECG was normal, and the patient denied any history of apparent myocardial infarction. Levodopa-carbidopa therapy was commenced at an initial dose of $100 \mathrm{mg}$ daily and subsequently increased to $200 \mathrm{mg}$ daily, following which his gait disturbance and hand immobility were resolved.

\section{Discussion}

In their case report of Parkinson's disease associated with type I Gaucher's disease, Tayebi et al (1) described a crossover between the glucocerebrocidase gene (GBA) and metaxin gene. The metaxin gene is involved in mitochondrial function, which may be altered in Parkinson's disease. Aharon-Peretz et al (2) reported that $31.3 \%$ of cases with Parkinson's disease exhibit genetic susceptibility. These studies suggest a common genetic basis for both Gaucher's disease and Parkinson's disease. To date, no anomaly in the

\footnotetext{
${ }^{1}$ Department of Neurology, Saitama Medical University, Saitama, ${ }^{2}$ Department of Hematology, Saitama Medical University, Saitama and ${ }^{3}$ Positron Medical Center, Tokyo Metropolitan Institute of Gerontology, Tokyo

Received for publication February 21, 2006; Accepted for publication June 20, 2006

Correspondence to Dr. Kaori Itokawa, Department of Neurology, Saitama Medical School, 38 Moro Hongo, Moroyama-cho, Iruma-gun, Saitama $350-0495$
} 


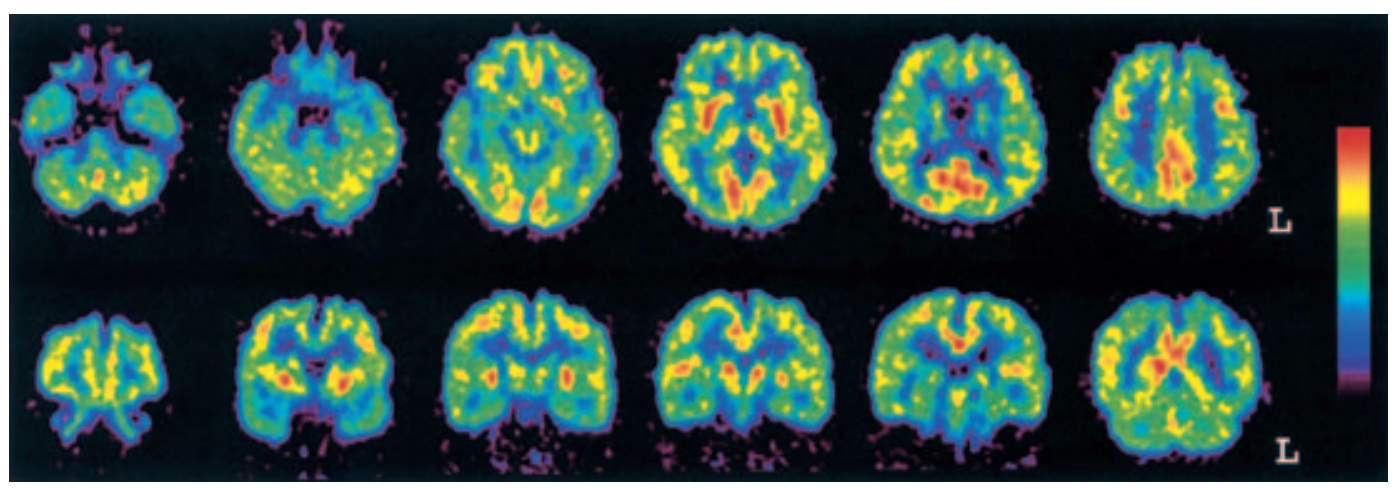

Figure 1. [F-18] FDG PET shows hypometabolism in the frontal and parietal cortices, but metabolism in the striatum is well preserved.

a

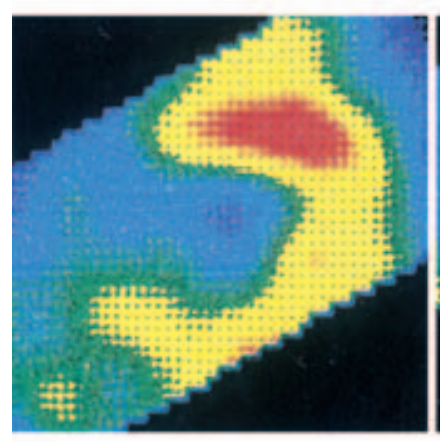

b
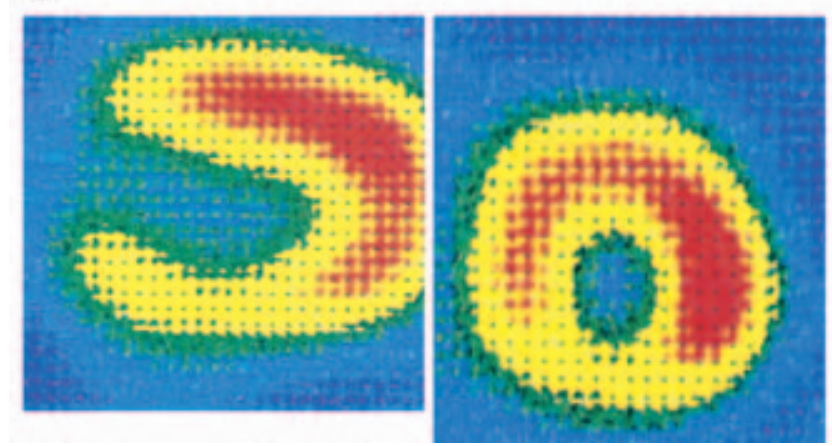

Figure 2. MIBG myocardial SPECT shows persistent low uptake in the infero-posterior area (a); however, [Tc-99m] tetrofosmin SPECT also reveals low uptake inthe sptum and inferior areas (b), suggesting that reduced MIBG uptake is due to cardiac infarction rather than sympathetic denervation. $\mathrm{H} / \mathrm{M}$ ratio is $\mathbf{1 . 8 4}$.

GBA/metaxin gene has been identified in any form of hereditary parkinsonism, but we do not consider the coexistence of Gaucher's disease and Parkinson's disease to be a mere chance association.

However, the clinical features of Parkinson's disease in type I Gaucher's disease differ somewhat from those of typical Parkinson's disease (1-7). In most published cases, the age at onset of parkinsonism was younger than that seen in typical Parkinson's disease, resting tremor was not evident, and the effect of levodopa was relatively poor. However, further clinical investigations were not described in these articles. In the present case, parkinsonism responded well to levodopa therapy, and [F-18] fluorodeoxyglucose (FDG) PET revealed that function of the striatum, a postsynaptic structure of the nigro-striatal dopaminergic pathway, was well preserved. These findings are identical to those of idiopathic Parkinson's disease.

However, onset as early as the 5th decade of life, absence of resting tremor, and relative preservation of myocardial MIBG uptake are not typical features of idiopathic Parkinson's disease. In particular, MIBG myocardial scintigraphy has been established as a useful tool to distinguish idiopathic Parkinson's disease from other parkinsonian syndromes such as multiple system atrophy and progressive supranuclear palsy. Myocardial uptake of MIBG is completely absent, indicating cardiac sympathetic denervation, in the majority of cases of idiopathic Parkinson's disease, even in the earlier stages, while it is well-preserved in other parkinsonian syndromes (8-11). In the present case, apart from a partial defect of MIBG uptake probably due to old myocardial infarction, MIBG scintigraphy did not indicate a reduced uptake. Normal uptake of MIBG in the present case may represent a lack of Levy body pathology, since MIBG uptake is also preserved in autosomal recessive juvenile parkinsonism with PARK2 anomaly (10).

It is noteworthy that the clinical features of parkinsonism in Gaucher's disease differ distinctly from those of typical Parkinson's disease. While we do not consider the coexistence of Gaucher's disease and Parkinson's disease to represent a mere chance association, it is noteworthy that the clinical features of such cases differ distinctly from those of typical Parkinson's disease. Gaucher's diseaserelated parkinsonism may account for a small number of cases of Parkinson's disease. 


\section{References}

1. Tayebi N, Callahan M, Madike V, et al. Gaucher disease and parkinsonism: a phenotypic and genotypic characterization. Mol Genet Metab 73: 313-321, 2001.

2. Aharon-Peretz J, Rosenbaum H, Gershoni-Baruch R. Mutations in the glucocerebrosidase gene and Parkinson's disease in Ashkenazi Jews. N Engl J Med 351: 1972-1977, 2004.

3. Neudorfer O, Giladi N, Elstein D, et al. Occurrence of Parkinson's syndrome in type I Gaucher disease. Q J M 89: 691-694, 1996.

4. Varkonyi J, Simon Z, Soos K, Poros A. Gaucher disease type I complicated with Parkinson's syndrome. Haematologia 32: 271275, 2002.

5. Macaczka M, Rucinska M, Skotnicki AB, Jurczal W. Parkinson's syndrome preceding clinical manifestation of Gaucher's disease. Am J Hematol 61: 216-217, 1999.

6. McKeran RO, Bradbury P, Taylor D, Stern G. Neurological involvement in type I (adult) Gaucher's disease. J Neurol Neurosurg
Psychiatry 48: 172-175, 1985.

7. Neil JF, Glew RH, Peters SP. Familial psychosis and diverse neurologic abnormalities in adult-onset Gaucher's disease. Arch Neurol 36: 95-99, 1979.

8. Yoshita M. Differentiation of idiopathic Parkinson's disease from striatonigral degeneration and progressive supranuclear palsy using iodine-123 metaiodobenzylguanidine myocardial scintigraphy. J Neurol Sci 155: 60-67, 1998.

9. Orimo S, Ozawa E, Nakade S, Sugimoto T, Mizusawa H. ${ }^{123} \mathrm{I}-$ metaiodobenzylguanidine myocardial scintigraphy. J Neurol Neurosurg Psychiatry 67: 189-194, 1999.

10. Orimo $S$, Amino $T$, Yokochi $M$, et al. Preserved cardiac sympathetic nerve accounts for normal cardiac uptake of MIBG in PARK2. Mov Disord 20: 1350-1353, 2005.

11. Druschky A, Hilz MJ, Platsch G, et al. Differentiation of Parkinson's disease and multiple system atrophy in early disease by means of I-123-MIBG-SPECT. J Neurol Sci 175: 3-12, 2000.

(C) 2006 The Japanese Society of Internal Medicine http://www.naika.or.jp/imindex.html 